\title{
Rise Time of Pulsed Parametric Oscillators
}

\author{
JAMES E. PEARSON, STUdent MEMber, IEee, U. GANIEL, AND AMNON YARIV, Fellow, IEeE
}

\begin{abstract}
General relations are derived that describe the rise time of the output power of parametric oscillators driven by a timedependent pump. Both singly resonant and doubly resonant oscillators are discussed. Results of computer calculations using a Gaussian time envelope for the pump pulse are presented and, in the case of the doubly resonant oscillator, are compared to predictions of a steady-state analysis. Agreement between calculated rise times and published values is good.
\end{abstract}

\section{Introduction}

A PARAMETER of great practical importance in the design and operation of a parametric oscillator is the rise time of the output power of such a device. In CW operation the rise time is a measure of the time the oscillator requires to reach steady state in response to perturbations. In pulsed operation, the rise time may limit the amount of power transferred from the pump to the signal and idler fields, possibly even preventing the oscillator from turning on.

Previous calculations of the rise time of parametric oscillators [1], [2] have assumed a constant pump power and have neglected spatial variations of the oscillator signal and idler fields. Such results are not strictly applicable for pulsed oscillators and are inadequate to describe the rise time of a singly resonant oscillator (SRO) where the nonresonant field has a strong spatial variation. In addition, because of the large pump power requirements of most oscillators, the pulsed parametric oscillator driven by a Q-switched laser has emerged as the most practical form of this device [3]-[14].

The use of a pulsed pump, however, changes the whole nature of the oscillator operation, affecting both the effective pump threshold and the power conversion efficiency from pump to signal and idler. With a pulsed pump it is possible for the peak power of the pump pulse to exceed the $\mathrm{CW}$ threshold by a large factor and yet result in a negligible power transfer to the oscillator fields. This will happen if the buildup time of the resonant signal (or idler) power is comparable to the time the pump power remains above threshold.

We undertake in this paper the task of calculating the rise time of pulsed singly and doubly resonant oscillators $(\mathrm{DRO})$. The results are then used to derive

Manuscript received September 10, 1971: revised November 24,1971 . This work was supported in part by a grant from the General Dynamics Corporation.

J. E. Pearson and A. Yariv are with the California Institute of Technology, Pasadena, Calif. 91109.

U. Ganiel is with the California Institute of Technology, Pasadena, Calif. 91109, on leave from the Weizmann Institute of Science, Rehoyot, Israel. relationships that must be obeyed by the various oscillator parameters to ensure efficient power conversion. Numerical results using a Gaussian time envelope for the pump pulse are presented and significant differences are found between these results and rise-time values computed using a square pump pulse and a steady-state analysis [1], [2]. Finally, experimental rise-time values found in the literature are compared to computed values and good agreement is found,

\section{Nonlinear Interaction Equations}

In the most general case, the fields involved in a parametric oscillator are functions of both space and time. For the rise-time calculations to be considered here, however, the pump field of angular frequency $\omega_{3}$ is assumed to be unaffected by the parametric interaction (nondepleted pump) so that only the signal $\left(\omega_{1}\right)$ and idler $\left(\omega_{2}\right)$ fields will be considered. The pump field is also assumed to be monochromatic and to have a "slowly varying" time envelope characteristic of a Q-switched laser pulse.

Using these assumptions in Maxwell's equations with nonlinear polarization terms, requiring $\omega_{3}=\omega_{1}+\omega_{2}$ and making the usual slowly varying envelope approximations gives

$\frac{\partial E_{1}}{\partial z}+\frac{n_{1}}{c} \frac{\partial E_{1}}{\partial t}+a_{1} E_{1}=-i K_{1} \exp [i \Delta \phi] \exp [-i \Delta k z] E_{2} E_{3}$

$\frac{\partial E_{2}}{\partial z}+\frac{n_{2}}{c} \frac{\partial E_{2}}{\partial t}+a_{2} E_{2}=-i K_{2} \exp [i \Delta \phi] \exp [-i \Delta k z] E_{1} E_{3}$

where $E_{j}=E_{j}(z, t)$ is the real traveling-wave field amplitude and

$$
\begin{aligned}
\Delta \phi & =\phi_{3}-\phi_{2}-\phi_{1}, & \Delta k & =k_{3}-k_{2}-k_{1} \\
K_{i} & =\frac{\omega_{i} d_{e} M}{2 c \epsilon_{0} n_{i}}, & j & =1,2 .
\end{aligned}
$$

The effective nonlinear coefficient [15], [16] is $d_{\theta}$, the index of refraction at $\omega_{j}$ is $n_{j}$, and the constant $M$ results from integrating the equations over the transverse coordinates. The value of $M$ for Gaussian beams and a near-field approximation is

$$
\begin{aligned}
M=2 \sqrt{2 / \pi} W_{1} W_{2} W_{3} /\left[\left(W_{1} W_{2}\right)^{2}\right. & \\
& \left.+\left(W_{1} W_{3}\right)^{2}+\left(W_{2} W_{3}\right)^{2}\right] .
\end{aligned}
$$

Double refraction effects can also be included in $M$ since they influence the threshold of a parametric oscillator [15]. The definition of $E_{j}$ used in (1) and (2) gives 
the time averaged power as $P_{j}=\epsilon_{o} n_{j} c E^{2}{ }_{j}\left(z_{1} t\right) / 2$. For resonant fields, $a_{j}=\alpha_{j} / L_{j}^{\prime}$ where $\alpha_{j}$ is the fractional round-trip field loss or one-way power loss and $L_{j}^{\prime}$ is the optical length of the cavity at $\omega_{j}$. For nonabsorbing highoptical-quality crystals, $a_{j}$ can be set equal to zero for nonresonant fields; the absence of feedback is then accounted for by the boundary conditions.

The pulsed nature of the pump field is accounted for by writing it as

$$
E_{3}(z, t)=E_{3 p} f(t)
$$

where $E_{3 p}$ is the peak value of the field at $\omega_{3}$ and $f(t)$ has a peak value of unity. Note that the pump envelope is assumed independent of $z$. The envelope function $f(t)$ will later be taken to the Gaussian but for the moment is left arbitrary. Defining $t=0$ as the time when the oscillator first reaches threshold, a pumping ratio $N$ can be defined by

$$
N=\frac{P_{3 p}}{P_{3 T}}=\frac{E_{3 p}{ }^{2}}{E_{3 T}}=\frac{1}{f^{2}(0)} .
$$

The threshold ${ }^{1}$ for oscillation is $P_{3 T}$ and $N$ is the factor by which the pump peak power exceeds threshold. The quantity $P_{3 T}$ as defined here is what is usually considered to be the $\mathrm{CW}$ threshold [15] (gain $=$ loss) and may differ considerably from the experimentally observed threshold (minimum peak pump power for oscillation to occur). The separate cases of singly resonant and doubly resonant oscillators will now be considered.

\section{Singly Resonant Oscillator}

In this case the signal field $\left(\omega_{1}\right)$ is taken to be resonant and its amplitude assumed independent of $z$ so $\partial E_{1} / \partial z$ $\approx 0$. There is no feedback at the idler frequency so that for a lossless crystal $a_{2} \approx 0$ and $E_{2}(0, t)=0$. Exact phase matching is assumed $(\Delta k=0)$ and the relative phase $\Delta \phi$ is assumed to be that which yields maximum conversion of pump energy into the signal and idler fields. For $\Delta k=0$, the condition is $\Delta \phi=\pi / 2$. Equation (1) is then integrated over the cavity length $L$, noting that $n_{1}=1$ and $K_{1}=0$ except over the length $l$ of the nonlinear crystal and the result combined with (2) to give a single integral equation for $E_{1}(t)$. Using the boundary conditions $E_{2}(0, t)=0$ and $E_{1}(t=n l / c)$ $\equiv E_{10}$, the result is

$$
\begin{aligned}
R_{1}{ }^{1 / 2}(t)=\exp \left[-\left(\frac{c \alpha_{1} t}{L^{\prime}}\right)\right]\left[\exp \left(\frac{n \alpha_{1} l}{L^{\prime}}\right)+I(t)\right] & , \\
t & \geq \frac{n l}{c}
\end{aligned}
$$

where

$$
R_{1}(t)=E_{1}^{2}(t) / E_{10}{ }^{2}=P_{1}(t) / P_{10}
$$

\footnotetext{
${ }^{1}$ For the SRO it is straightforward to show that $E^{2}{ }_{3 r}=2 \alpha_{1} /$ $K_{1} l^{2}$. For the DRO the threshold condition is $E^{2_{3 x}}=\alpha^{2} / K_{1} K_{2} l^{2}$ for $\alpha_{1}=\alpha_{2}$.
}

and

$$
\begin{array}{r}
I(t) \equiv \frac{2 c^{3} \alpha_{1} N}{n^{2} l^{2} L^{\prime}} \int_{n l / c}^{t} \int_{x-(n l / c)}^{x} \exp \left[\alpha_{1} \frac{c}{L^{\prime}} x\right] f(x) f(y) \\
\cdot\left(\frac{n l}{c}+y-x\right) R_{1}^{1 / 2}(y) d y d x .
\end{array}
$$

The optical length of the cavity is $L^{\prime}$, assumed equal at $\omega_{1}$ and $\omega_{2}, n=n_{1} \simeq n_{2}$, and $\alpha_{1}$ is the one-way fractional power loss at $\omega_{1}$.

Note that the initial condition on $E_{1}$ has been specified at $t=n l / c$ rather than at $t=0$. We have thus assumed $E_{1} \simeq E_{10}$ for the first transit time through the crystal. This procedure ensures that $E_{2}$ is nonzero throughout the crystal for all times $t$ under consideration and allows the integral over $l$ to be performed. Because of the slow growth rate of the resonant signal near threshold, this definition of the initial condition on $E_{1}$ introduces negligible error and eliminates the cumbersome mathematical formalism that results from assuming $E_{2}(0, t)=0$ and thus $E_{2}(l, t)=0$ for times less than $n l / c^{2}$

The time dependence of the pump field is now assumed to be Gaussian, a form that closely approximates the output pulse of a $Q$-switched laser. The envelope function $f(t)$ is thus

$$
f(t)=\exp \left[-\left(t-\tau_{T}\right)^{2} / a^{2}\right]
$$

where

$$
a^{2}=\frac{\tau_{p}^{2}}{2 \ln (2)}
$$

and $\tau_{p}$ is defined as the full width at half maximum of the pump pulse intensity. The quantity $\tau_{T}$ is the time between the oscillator threshold level $(t=0)$ and the peak of the pump pulse and can be written as

$$
\tau_{T}^{2}=\frac{a^{2} \ln (N)}{2}=\frac{\tau_{\nu}{ }^{2} \ln (N)}{4 \ln (2)}
$$

with $N$ being defined by (4). The relationships between the various quantities are illustrated in Fig. 1(a).

The rise time of a parametric oscillator can be defined in a number of ways. A convenient operational approach is to define the rise time $\tau_{R}$ as the time required for the signal output power to reach a predetermined value starting from the moment the pump reaches threshold. Defining $P_{1 e}$ as the signal output power and assuming the only loss is due to output coupling, this definition of $\tau_{R}$ can be written as

$$
P_{1 e}\left(\tau_{R}\right) \equiv \alpha_{1} P_{1}\left(\tau_{R}\right)=\text { constant }
$$

Since the initial internal signal power $P_{1}(0) \equiv P_{10}$ is due to spontaneous parametric fluorescence driven by the threshold pump power, we have $P_{10} \propto P_{3 T} / \alpha_{1}$. However, for the SRO, ${ }^{1} P_{3 T} \propto \alpha_{1}$ so that $P_{10}$ is independent of the

2 This condition is not strictly true because some parametric fluorescence at $\omega_{s}$ is always present at the output of the nonlinear crystal. 


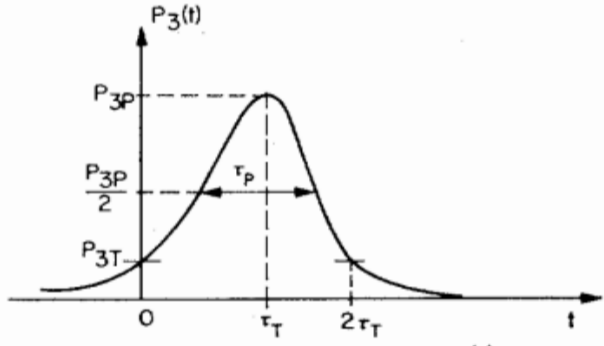

(a)

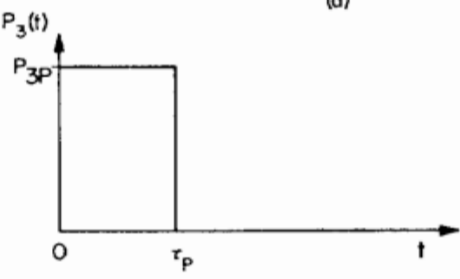

(b)

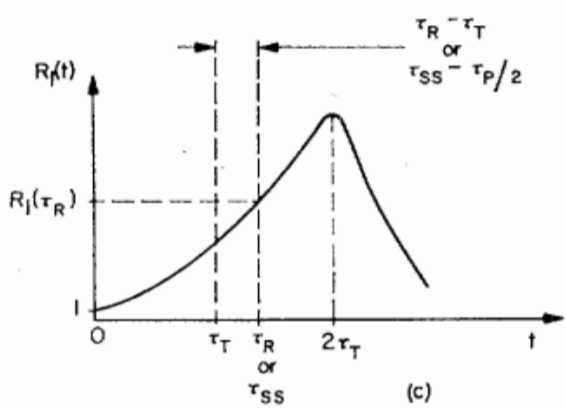

Fig. 1. (a) Gaussian pump pulse defining the quantities $\tau_{r}, P_{3 T}$ and $P_{3 p}$. Note $t=0$ is defined by the oscillator threshold level. (b) Square-pulse approximation to the Gaussian used with steady-state equations for the DRO. (c) Qualitative plot of $R_{1}(t)=P_{1}(t) / P_{10}$ showing the relationship between $\tau_{R}$ and $\tau_{\text {r. }}$. Note that in the undepleted pump approximation used here the signal power continues to grow until the pump falls below threshold $\left(t=2 \tau_{T}\right)$.

coupling loss $\alpha_{1} .{ }^{3}$ Consequently, the constant appearing in (9) can be replaced by $r P_{10}$ where $r$ is some constant. The rise time $\tau_{R}$ is now defined by $\alpha_{1} P_{1}\left(\tau_{R}\right)=r P_{10}$ or using $R_{1}(t) \equiv P_{1}(t) / P_{10}$

$$
R_{1}\left(\tau_{R}\right)=r / \alpha_{1} .
$$

Once a value for $r$ is chosen, (10) is used with (5) and (6) to find $\tau_{R}$.

Although in the sense defined here the oscillator is not on until (10) is satisfied, the oscillator is always on in the sense of the pump being above the CW threshold for all times in the range $t=0$ to $t=2 \tau_{T}$. The signal and idler fields experience a net round-trip gain and thus grow in amplitude during this time period. The value of $r$ chosen for calculating rise time is arbitrary, but fortunately, because of the "explosive" nature of the signal buildup, $\tau_{R}$ is not a strong function of the ratio $r / \alpha_{1}$. A change of two orders of magnitude in $r$ results in a change of $\tau_{R}$ by only about 10 percent.

For experimental comparisons a useful parameter

${ }^{3}$ This conclusion is true only if the feedback at the signal (and idler in the case of the DRO) is small enough so that the effective input to the spontaneous fluorescence remains approximately one photon per mode [17].

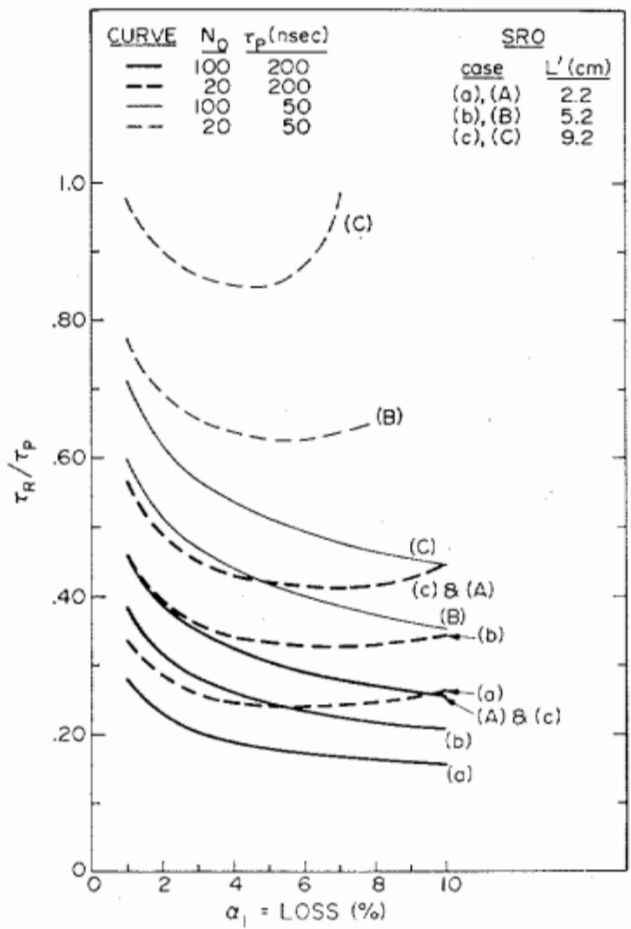

Fig. 2. SRO absolute rise time divided by pump pulsewidth versus resonant loss for various values of cavity optical length and normalized pump ratio. $\tau_{p}=200 \mathrm{~ns}$-heavy lines or 50 ns-light lines and $r=10^{8}$.

is the quantity $P_{3 P} /\left(P_{3 T} / \alpha_{1}\right)=N \alpha_{1}$ since the peak pump power is usually fixed and knowledge of the crystal parameters allows computation of the quality $P_{3 T} / \alpha_{1}$. Fixing the quantity $N \alpha_{1}$ is equivalent to fixing the peak pump power and allows the effect of different cavity losses on rise time to be predicted. For all the numerical results of this paper, therefore, a normalized pump ratio $N_{0}$ has been used instead of $N$ as defined by (4). For the SRO, $N_{0}$ is defined as the pumping ratio multiplied by the resonant loss in percent:

$$
N_{0}=N\left(100 \alpha_{1}\right) .
$$

Equation (5) cannot be solved in closed form for $R_{1}(t)$ but can be solved numerically. Using digital computer techniques, the rise time for a singly resonant oscillator has been computed using (5). The results for two values of $\tau_{p}$ are shown in Figs. 2-4. Figs. 2 and 3 plot the ratio of the rise time to the pump pulsewidth. The ordinate axis in Fig. 4 is the time between the peak of the pump pulse and the moment the ocsillator turns ov [see Fig. 1 (c) ] divided by $\tau_{p}$. This is the most convenient to measure experimentally and so is the most useful comparison to experiment. A value of $r=10^{8}$ has been used in all calculations. For an initial internal power $P_{10}=10^{-10} \mathrm{~W}$, this choice of $r$ corresponds to an external power of $10^{-2}$ $\mathrm{W}$ and for $\alpha_{1}=1$ percent an internal power of $1 \mathrm{~W}$.

\section{Doubly Resonant Oscillator}

The simplest case to consider for the DRO is the one for equal losses at $\omega_{1}$ and $\omega_{2}$. This is the usual case ex- 


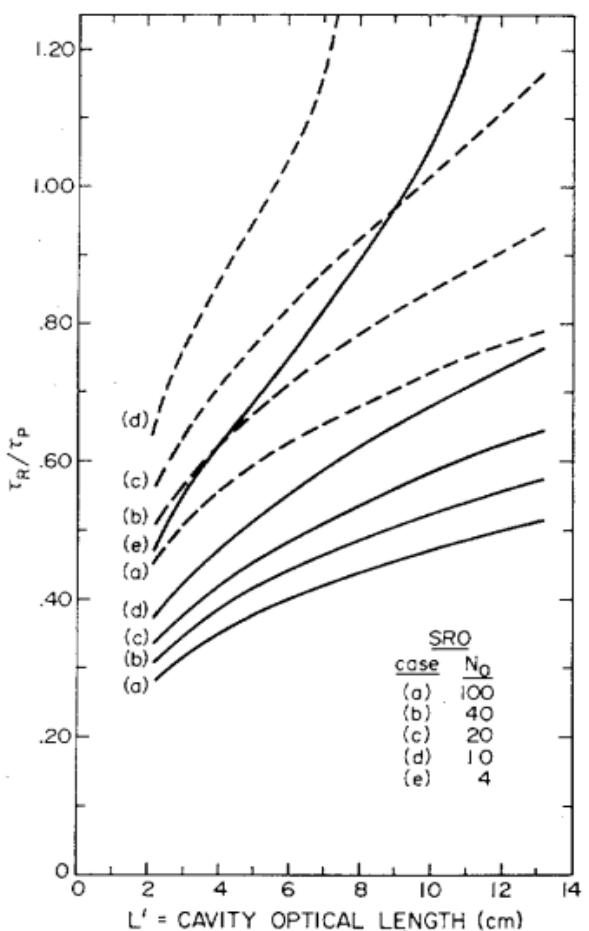

Fig. 3. SRO absolute rise time divided by pump pulsewidth as a function of cavity optical length for various normalized pump ratios. $\tau_{p}=200 \mathrm{~ns}-$ solid lines or $50 \mathrm{~ns}-$ broken lines; $\alpha_{1}=1$ percent, $r=10^{8}$.

perimentally and will be the only one treated here. Setting $\partial / \partial z=0$ in (1) and (2), integrating each over the cavity length, and solving the resulting time-dependent equations with initial conditions $E_{1}(0)=E_{10}$ and $E_{2}(0)$ $=E_{20}$ gives $E_{1}(t)$ as

$$
\begin{aligned}
E_{1}(t)= & \frac{1}{2}\left[\left(E_{10}+\sqrt{\left({K^{\prime}}_{1} / K^{\prime}{ }_{2}\right)} E_{20}\right) \exp \left[t s_{+}(t)\right]\right. \\
& \left.+\left(E_{10}-\sqrt{\left({K^{\prime}}_{1} / K^{\prime}{ }_{2}\right)} E_{20}\right) \exp \left[t s_{-}(t)\right]\right]
\end{aligned}
$$

where $\beta=c \alpha / L^{\prime}, K^{\prime}{ }_{j}=K_{j} \sin (\Delta k l / 2) /(\Delta k l / 2)$ and

$$
t s_{ \pm}(t)=\beta\left[N^{1 / 2} g(t)-t\right] .
$$

The relative phase has been chosen as $\Delta \phi=\Delta k l / 2+$ $(\pi / 2)$ to maximize the interaction and the function $g(t)$ is defined by

$g(t)=\int_{0}^{t} f(t) d t=\frac{a \sqrt{\pi}}{2}\left[\operatorname{erf}\left(\frac{t-\tau_{T}}{a}\right)+\operatorname{erf}\left(\frac{\tau_{T}}{a}\right)\right]$.

The integral in (14) has been evaluated for $f(t)$ as defined by (7) and (8) and

$$
\operatorname{erf}(x)=\frac{2}{\sqrt{\pi}} \int_{0}^{x} e^{-u^{2}} d u .
$$

For the DRO as well as the SRO, the initial values of the resonant fields are found from the parametric fluorescence power inside the resonator at threshold and within the bandwidth of the oscillator [2], [17]. Since these values are many orders of magnitude below the

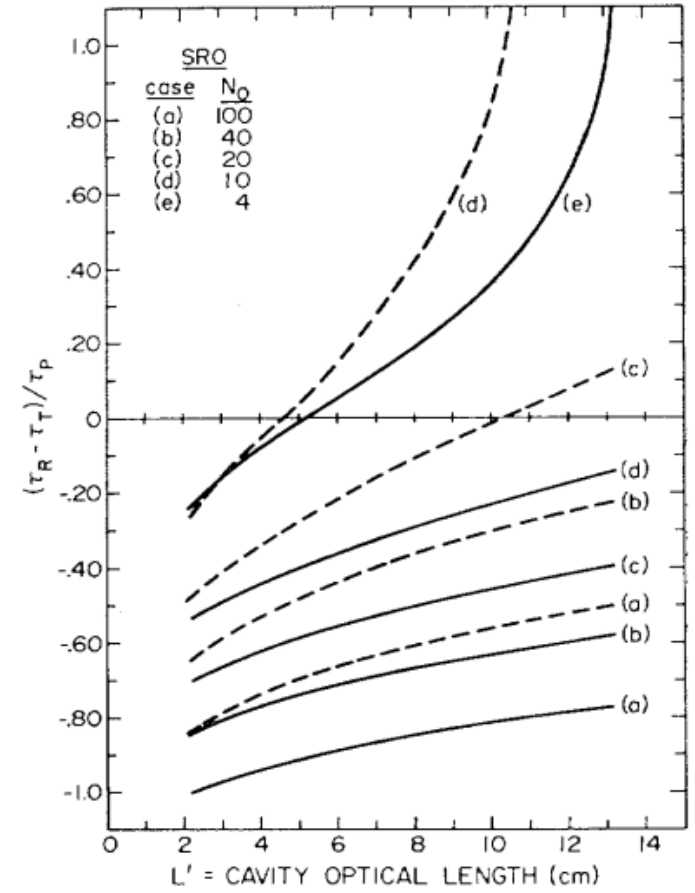

Fig. 4. SRO rise time relative to the peak of the Gaussian pump pulse divided by $\tau_{p}$ plotted as a function of cavity optical length. $\tau_{p}=200 \mathrm{~ns}$-solid lines or $50 \mathrm{~ns}$-broken lines; $\alpha_{1}=$ 1 percent, $r=10^{8}$.

oscillator operation level, only the rapidly growing part of (12) will contribute to the field at $\omega_{1}$. Neglecting the second term of (12), the power output at $\omega_{1}$ can be written as

$P_{1}(t)=\frac{1}{4}(1+\gamma)^{2} P_{1}(0) \exp [2 t s(t)]=P_{10} \exp [2 t s(t)]$ where from (12) and [17],

$$
\gamma=\sqrt{\frac{K_{1}}{K_{2}}} \frac{E_{20}}{E_{10}}=\left[\frac{\omega_{1} P_{2}(0)}{\omega_{2} P_{1}(0)}\right]^{1 / 2}=\frac{\omega_{2} n_{2}}{\omega_{1} n_{1}}
$$

and

$$
P_{10}=\frac{(1+\gamma)^{2}}{4} P_{1}(0) .
$$

$P_{10}$ is the initial input to the signal oscillator mode.

Contrary to the case of the SRO, $P_{\mathbf{1 0}}$ is not independent of the loss of the DRO because now $P_{3 T} \propto \alpha^{2}$. As a result, $P_{10} / \alpha$ is a constant. Defining the rise time in the same way as for the SRO, the constant in (9) can be replaced by $r P_{10} / \alpha$ for the DRO. The rise time $\tau_{I R}$ is thus defined by the following equation:

$$
R_{1}\left(\tau_{R}\right) \equiv \frac{P_{\mathrm{l}}\left(\tau_{R}\right)}{P_{10}}=\frac{r}{\alpha^{2}} .
$$

Using (16), (13) and (15) give

$$
\tau_{R} s\left(\tau_{R}\right)=\frac{c \alpha}{L^{\prime}}\left(N^{1 / 2} g\left(\tau_{R}\right)-\tau_{R}\right)=\frac{\ln \left(r / \alpha^{2}\right)}{2} .
$$

Note that the ratio $r / \alpha^{2}$ enters as the argument of a 


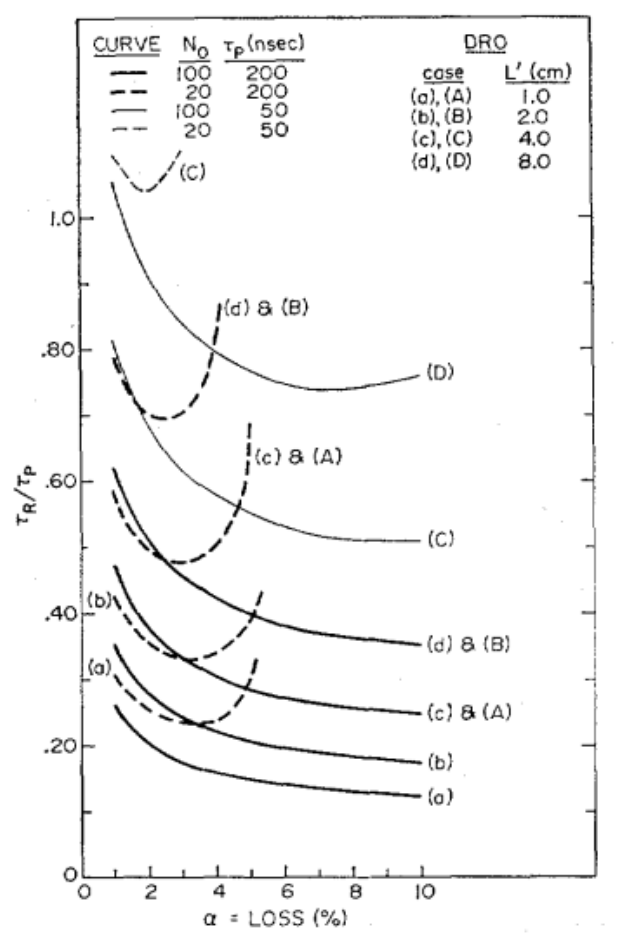

Fig. 5. DRO absolute rise time divided by pump pulsewidth versus resonant loss for various values of cavity optical length and normalized pumping ratio. $\tau_{p}=200$ ns-heavy lines or 50 ns-light lines; $r=10^{8}$.

logarithm, so that as in the case of the SRO, the rise time is not strongly dependent on the value chosen for $r$.

As noted in the Introduction, earlier calculations [1], [2] of the doubly resonant parametric oscillator rise time have assumed a constant pump power. This result can be found by setting $f(t)=1$ so $g(t)=t$; (17) then gives

$$
\tau_{s s}=\frac{L^{\prime} \ln \left(r_{s s} / \alpha^{2}\right)}{2 c \alpha\left(N^{1 / 2}-1\right)} .
$$

$r_{s s} / \alpha^{2}$ is the ratio of the external cavity steady-state power to the initial internal power and $\tau_{s s}$ is the time required to reach steady state. This result has previously been derived by Kreuzer [1] and by Byer [2] using a slightly different definition of rise time. One possible way of using (18) to predict the rise time of a pulsed oscillator is to assume the pump pulse is a square pulse of width $\tau_{p}$ as shown in Fig. 1(b). The magnitude of the square pulse is taken to be the same as the peak of the Gaussian pulse defining $\tau_{p}$. For the sake of comparison, the predictions obtained using this quasi-steady-state approach are also shown as light curves in Figs. 6 and 7 .

Results for the DRO for two values of $\tau_{p}$ are shown in Figs. 5-7. Fig. 5 shows the effect of losses on DRO rise time and Fig. 6 gives the ratio $\tau_{R} / \tau_{p}$ (or $\tau_{s s} / \tau_{p}$ ) as a function of cavity length for different pump ratios. Fig. 7 plots the quantity $\left(\tau_{R}-\tau_{T}\right) / \tau_{p}$ or $\left(\tau_{s s}-\tau_{p} / 2\right) / \tau_{p}$. The value $r=10^{8}$ has been used throughout. As in the case of the SRO, a normalized pumping parameter $N_{0}$, which

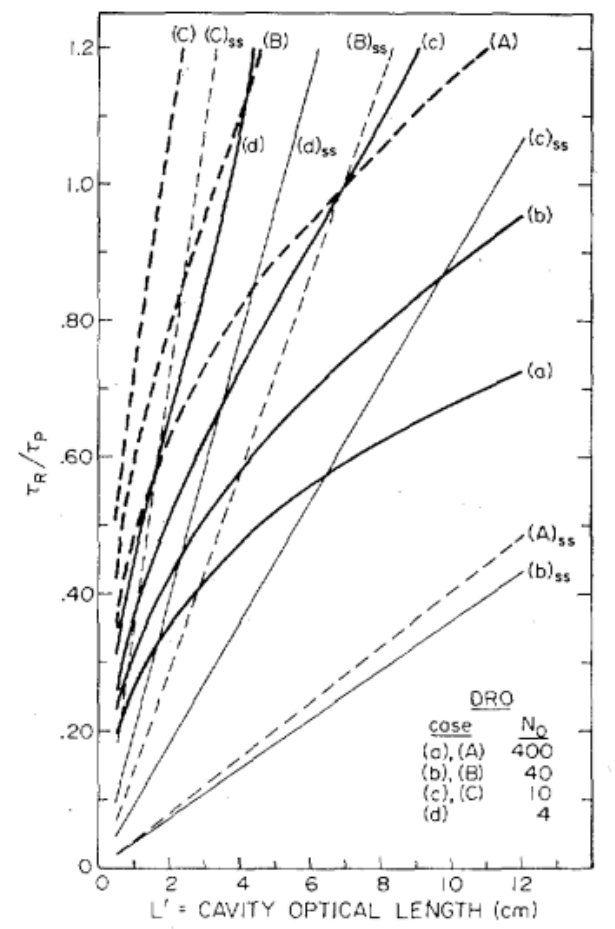

Fig. 6. DRO absolute rise time divided by pump pulsewidth as a function of cavity optical length for various normalized pump ratios. $\tau_{p}=200$ ns-solid lines or $50 \mathrm{ns-broken} \mathrm{lines;} \alpha=1$ percent, $r=10^{8}$. Light lines are results using the steady-state formula (18).

is independent of $\alpha$, is used in the figures. For the case of the DRO, $N_{0}$ is given by

$$
\begin{aligned}
& N_{0}=N(100 \alpha)^{2} . \\
& \text { V. Discussion }
\end{aligned}
$$

A number of interesting results are apparent from the figures. Figs. 2 and 5 show that increasing cavity loss may actually decrease the rise time even though larger loss means larger oscillator threshold. This curious behavior results from the use of a Gaussian pump pulse. The Gaussian function $e^{-x^{2}}$ has the property that for $x^{2}>\frac{1}{2}$ its slope increases in magnitude as $x^{2}$ decreases. Since the rate of growth of the resonant fields is proportional to the amount by which the pump exceeds threshold, the oscillator fields will grow most rapidly when the pump ratio $P_{3}(t) / P_{3 T}$ grows most rapidly. Lower losses imply a lower oscillator threshold and thus the threshold pump level occurs further out on the "tail" of the Gaussian, where the pump ratio changes more slowly per unit time. Consequently the rise time can be longer for smaller losses. However, the time $\left(\tau_{R}-\tau_{T}\right)$ increases monotonically with increasing loss reflecting the fact that for larger losses, the pump is above threshold for a shorter total time.

As mentioned previously, the curves shown above were computed using the value $r=10^{\mathrm{s}}$. Rise-time values have also been computed using values of $r=10^{6}, 10^{10}$, and $10^{12}$. A change of two orders of magnitude in $r$ changes 


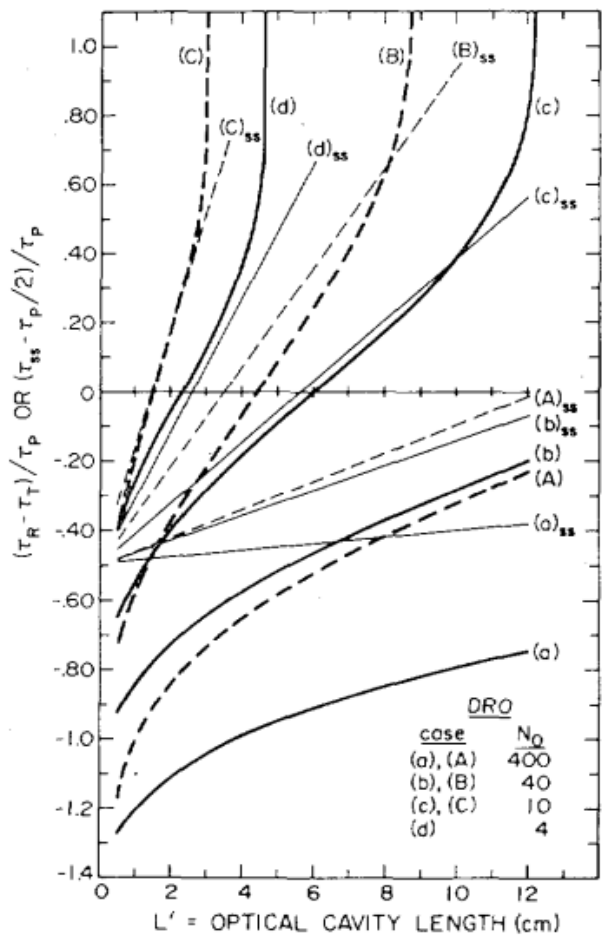

Fig. 7. DRO rise time relative to peak of Gaussian pump pulse divided by $\tau_{3}$ plotted as a function of cavity optical length. $\tau_{p}=200 \mathrm{~ns}-$ solid lines or $50 \mathrm{~ns}-$ broken lines; $\alpha=1$ percent, $r=10^{\mathrm{s}}$. Light lines are results using steady-state formula (18).

$\tau_{R}$ by roughly 10 percent. The only time the value of $r$ makes a significant difference in the calculated values of $\tau_{R}$ is when the parameters $\tau_{p}, L^{\prime}, \alpha$, and $N_{0}$ are such that the on condition can barely be achieved.

It is apparent from the figures that the rise time can be longer than the pump pulsewidth $\tau_{p}$. The important quantity, however, is $\tau_{R}$ compared to $\tau_{T}$ [see Fig. 1(c)]. If $\tau_{R}>2 \tau_{T}$, the oscillator will not turn on in the practical sense that the output power will never reach the preselected level. Inspection of (17) shows that the maximum value of $\tau_{R} s\left(\tau_{R}\right)$ occurs for $\tau_{R}=2 \tau_{T}$ at which point the pump power again crosses the threshold level [see Fig. 1(a)]. Use of this value for $\tau_{R}$ in (17) will define a minimum allowable pump ratio $N_{\min }$ given $\alpha$ and $L^{\prime}$ or, conversely, a maximum cavity optical length $L_{\max }^{\prime}$ given $N$ and $\alpha$. These extrema are given by the solutions to the following equations:

$$
e^{x^{2}} \operatorname{erf}(x)-\frac{2}{\sqrt{\pi}} x=\frac{L^{\prime} \ln \left(r / \alpha^{2}\right)}{2 \operatorname{co\alpha } \alpha \sqrt{\pi}}
$$

where

$$
x^{2}=\ln \left(N_{\min }\right) / 2
$$

and

$$
L_{\max }^{\prime}=\frac{2 c \alpha a \sqrt{\pi}}{\ln \left(r / \alpha^{2}\right)}\left[N^{1 / 2} \operatorname{erf}(y)-\frac{2}{\sqrt{\pi}} y\right]
$$

with $y=\ln (N) / 2$.

The corresponding quantities using the steady-state

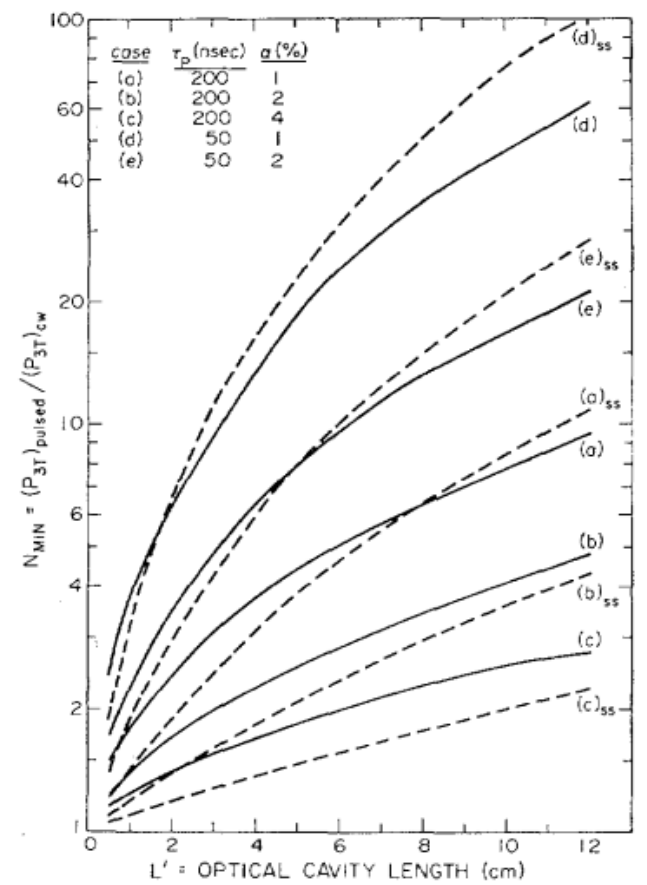

Fig. 8. Minimum pump ratio for a DRO to reach the condition $r=10^{8}$. Various cases of $\alpha$ and $\tau_{p}$ are shown versus cavity optical length. Dashed lines are results of the steady-state formula (22). The value $N_{\min }=1$ at $L^{\prime}=0$ corresponds to $\mathrm{CW}$ threshold.

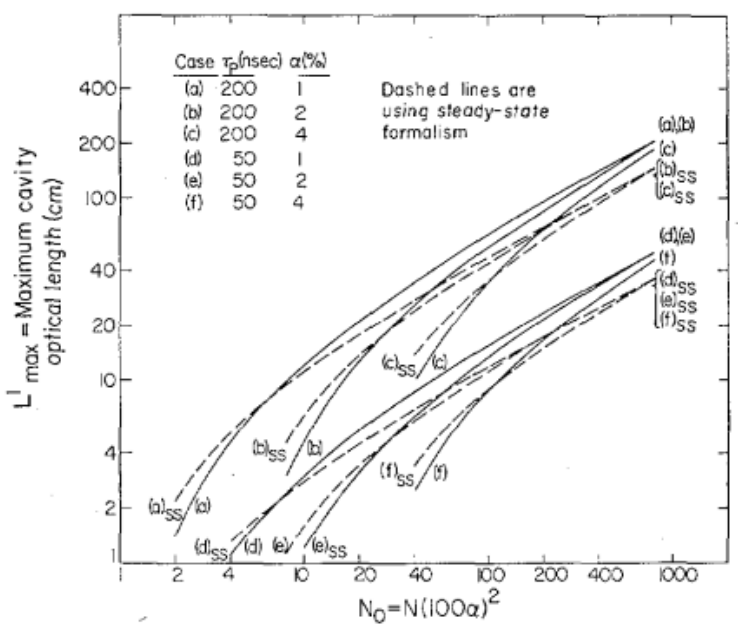

Fig. 9. Maximum cavity optical length for a DRO to reach the condition $r=10^{8}$. Various cases of $\alpha$ and $\tau_{p}$ are shown versus cavity optical length. Dashed lines are results of a steady-state formula $(23)$.

approximation of a square pulse and (18) are

$$
\begin{aligned}
\left(N_{\min }\right)_{s s} & =\left(\frac{L^{\prime} \ln \left(r / \alpha^{2}\right)}{2 c \alpha \tau_{p}}+1\right)^{2} \\
\left(L_{\max }^{\prime}\right)_{s s} & =\frac{2 c \alpha \tau_{p}\left(N^{1 / 2}-1\right)}{\ln \left(r / \alpha^{2}\right)} .
\end{aligned}
$$

Predictions of (20)-(23) are shown in Figs. 8 and 9 for two values of $\tau_{p}$. Note that the normalized pump ratio 
TABLE I

Relative Rise-Time Values Found in the Literature Compared to Calculated Values

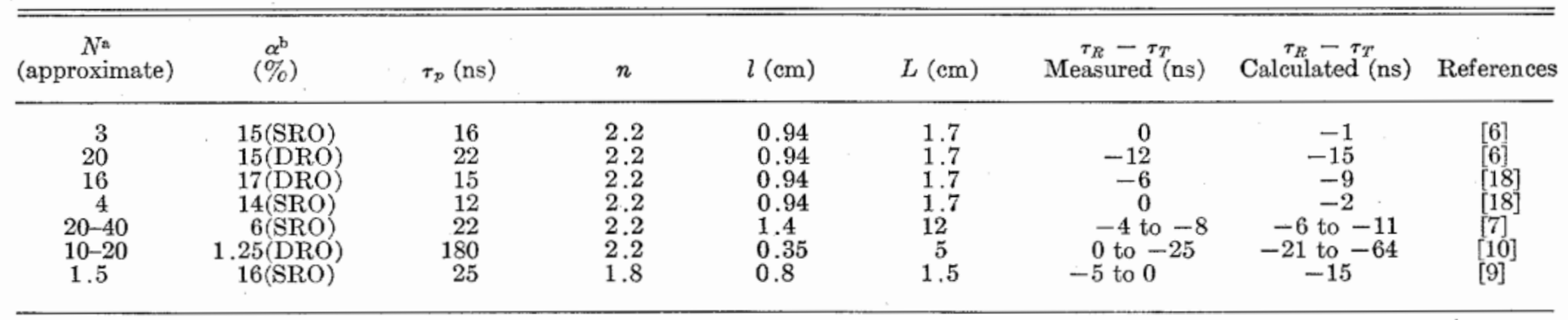

s Care must be taken in defining measured threshold for pulsed oscillators (see [18]). The results discussed in [18] have been used where possible in selecting the value of $N$ used here.

b In cases of the DRO where $\alpha_{1} \neq \alpha_{2}$, a value of $\alpha=\sqrt{\alpha_{1} \alpha_{2}}$ is assumed.

$N_{0}$ defined by (19) is used in Fig. 9. From the curves in Figs. 6-9 it can be seen that the results of the steadystate approximation are generally unreliable but might be used for a rough estimate.

The quantity $N_{\min }$ as defined by (20) and plotted in Fig. 8 is the factor by which the pulsed threshold is increased due to the finite oscillator rise time. Once the CW threshold has been calculated (using the results of [15], for example) and a cavity length chosen, the actual pulsed threshold is found by multiplying the $\mathrm{CW}$ result by $N_{\min }$ to obtain the threshold peak pump power. The pulsed oscillator threshold is thus defined by the minimum peak pump power for which the oscillator will turn on rather than the pump power that gives a net gain at the signal and idler frequencies. Note that if rise time were not an important consideration (i.e., CW case), $N_{\min }=1$. If the peak pump power is fixed ( $N_{0}$ is given), the maximum allowable cavity length can be found from Fig. 9. Note that for $L^{\prime}=L_{\max }^{\prime}$ or $N=N_{\min }$ the oscillator is driven at the "pulsed threshold" level.

From Figs. 2, 5, and 8 it is apparent that the effect of a finite oscillator rise time is often reduced for larger losses. It might appear advantageous to increase the losses in a given experimental situation. One should be careful in applying such logic, however, because the larger $\mathrm{CW}$ threshold resulting from increased loss could also prevent the oscillator from working. There is a definite tradeoff between increased threshold and reduced rise time and the optimum combination will have to be determined for each experimental situation.

Although the results shown in Figs. 8 and 9 are strictly applicable only to doubly resonant oscillators, they can also be used to give an estimate of the effect of rise time on SRO operation. For the SRO the abscissa of Fig. 9 should be $N_{0}=N\left(100 \alpha_{1}\right)$. It should be noted that the minimum pump values shown in Fig. 8 would apply for a perfectly adjusted oscillator pumped by a single-frequency pump. In a practical situation, a safety margin of about an order of magnitude in the pumping ratio would ensure ease of cavity alignment and stable reproducible operation, as well as ensuring significant energy conversion from the pump to the signal and idler fields.

\section{Comparison to Experiment and Conclusions}

Several experimental parametric oscillator results have been published and have included oscilloscope traces of the pump and signal output pulses. From these data, values of $\left(\tau_{R}-\tau_{T}\right)$ can be found and compared to theoretical predictions. From the experimental photographs, the time $\left(\tau_{R}-\tau_{T}\right)$ is defined by the onset of pump depletion or by the leading edge of the oscillator pulse if no depletion is evident. Table I sumarizes the result of a number of authors. The value of $r=10^{8}$ has been used for the predicted values.

The values of $N$ shown in Table I are quite approximate. Where enough information was supplied, the measured peak pump power and the theoretical $\mathrm{CW}$ threshold were used to find $N$. Otherwise $N$ was computed using the measured threshold power. Considering the approximate nature of the experimental data, the agreement with theory is considered reasonable.

The results presented here can be used to determine whether in a given experimental situation the signal power will approach the steady-state level corresponding to the peak pump power. The importance of this consideration is that as a result of the "explosive" nature of the signal buildup under pulsed excitation, failure to reach the quasi-steady-state level also implies a negligible conversion of pump power to signal power even though the peak pump power may exceed the CW threshold (gain $=$ loss) by a large factor. The parameters $N_{\min }$ and $L_{\max }^{\prime}$ can be used to set practical design limits on pulsed parametric oscillator systems.

\section{ACKNOWLEDGMENT}

The authors are grateful to Dr. V. Evtuhov for a critical reading of the manuscript and for many helpful discussions and suggestions.

\section{References}

[1] L. B. Kreuzer, "High-efficiency optical parametric oscillation-and power limiting in $\mathrm{LiNbO}_{3}, "$ Appl. Phys. Lett., vol. 13, pp. 57-59, 1968.

[2] R. L. Byer, "Parametric fluorescence and optical parametric oscillation," Ph.D. dissertation, Hansen Microwave Lab., Stanford Univ., Stanford, Calif., Rep. 1711, 1968.

[3] J. A. Giordmaine and R. C. Miller, "Optical parametric os- 
cillation in $\mathrm{LiNbO}_{3}$, in Physics of Quantum Electronics, P. O. Kelley, B. Lax, and P. E. Tannenwald, Eds. New York: McGraw-Hill, 1966, pp. 31-42.

[4] J. E. Bjorkholm, "Some spectral properties of doubly and singly resonant pulsed optical parametric oscillators," Appl. Phys, Lett., vol. 13, pp. 399-401, 1968.

[5] L. B. Kreuzer, "Ruby-laser-pumped optical parametric oscillator with electro-optic effect tuning," Appl. Phys. Lett., vol. 10 , pp. $336-338,1967$.

[6] J. E. Bjorkholm, "Efficient optical parametric oscillation using doubly and singly resonant cavities," Appl. Phys. Lett., vol. 13 , pp. 53-56, 1968 .

[7] J. Falk and J. E. Murray, "Single-cavity noncollinear optical parametric oscillation," Appl. Phys. Lett., vol. 14, pp. 245247,1969 .

[8] L. B. Kreuzer, "Single mode oscillation of a pulsed singly resonant optical parametric oscillator," Appl. Phys. Lett., vol. 15, pp. 263-265, 1969

[9] L. S. Goldberg, "Optical parametric oscillation in lithium iodate," Appl. Phys. Lett., vol. 17, pp. 489-491, 1970.

[10] E. O. Ammann, M. K. Oshman, J. D. Foster, and J. M. Yarborough, "Repetitively pumped optical parametric oscillator at 2.13," Appl. Phys. Lett., vol. 15, pp. 131-133, 1969.

[11] E. O. Ammann, J. M. Yarborough, M. K. Oshman, and
P. C. Montgomery, "Efficient internal optical parametri oscillation," Appl. Phys. Lett., vol. 16, pp. 309-312, 1970.

[12] S. A. Akhmananov et al., "Tunable parametric light generator with KDP crystal," JETP Lett., vol. 3, pp. 372-378 (trans. pp. 241-245), 1966.

[13] R. C. Miller and W. A. Nordland, "Tunable $\mathrm{LiNbO}_{3}$ optica oscillator with external mirrors," Appl. Phys. Lett., vol. 10, pp. 53-55, 1967.

[14] R. W. Wallace, "Stable, efficient optical parametric oscillators pumped with doubled Nd:YAG," Appl. Phys. Lelt., vol. 17 pp. 497-499, 1970

[15] G. D. Boyd and D. A. Kleinman, "Parametric interaction of focused light beams," J. Appl., Phys., vol. 39, pp. 3597-3639, 1968.

[16] J. E. Midwinter and J. Warner, "The effects of phasematching method and of uniaxial crystal symmetry on the polar distribution of second order nonlinear optical polarization," Brit. J. Appl. Phys., vol. 16, pp. 1135-1142, 1965.

[17] R. L. Byer and S. E. Harris, "Power and bandwith of spontaneous parametric emission," Phys. Rev., vol. 168, pp. $1064-1068,1968$.

[18] J. E. Bjorkholm, "Some effects of spatially nonuniform pumping in pulsed optical parametric oscillators," IEEE J. Quantum Electron., vol. QE-7, pp. 109-118, July 1971.

\title{
Influence of the Lifetime and Degeneracy of the ${ }^{4} \boldsymbol{I}_{11 / 2}$ Level on Nd-Glass Amplifiers
}

\author{
PETER C. MAGNANTE
}

\begin{abstract}
Gain saturation of an Nd-glass amplifier by $1.06-\mu \mathrm{m}$ light pulses is determined by the lifetimes and degeneracies of the two laser states as well as by thermalization rates among the components of the two multiplets to which the laser levels belong. Reported are measurements showing that although small signal gain was the same for amplified microsecond and picosecond pulses, comparable energy gain saturation occurred when the energy density of the mode-locked pulses was 1.9 times smaller than the amount for the microsecond pulses indicating terminal level filling with the shorter pulses. Our measurements indicate the terminal laser level, which is one of two unresolved groups in the ${ }^{4} I_{11 / 2}$ multiplet, has a lifetime greater than $50 \mathrm{~ns}$ and a degeneracy likely to be one or two. Pulse train distortion due to saturating amplification was meastred to find whether the thermalization rates among the levels of the laser multiplets were fast or slow compared to the $10^{-11}-\mathrm{s}$ pulses, but experimental uncertainties prohibit a determination.
\end{abstract}

\section{INTRoduction}

T HE ONSET of gain saturation in an Nd-glass amplifier occurs when an optical pulse traveling in the inverted medium becomes strong enough to change appreciably the population of the laser levels [1]. The rate of increase and extent of saturation depend not only on the optical pulse power density and duration,

Manuscript received September 29, 1971; revised December 14, 1971.

The author is with the Research Division, American Optical Corporation, Southbridge, Mass. 01550. but also on the lifetimes and degeneracies of the levels in the 1.06- $\mu \mathrm{m}$ transition.

The upper laser level shown in Fig. 1 is the lower lying component of the ${ }^{4} F_{3 / 2}$ pair with a several-hundred microsecond spontaneous emission lifetime. Not counting the sublevels that would be split by a magnetic field, the degeneracy of either member of the ${ }^{4} F_{3 / 2}$ multiplet is one. The terminal laser level is the lower lying of two groups in the six-fold degenerate ${ }^{4} I_{11 / 2}$ multiplet. Spectroscopically the components of either group in this manifold have been unresolved leaving the degeneracy of the terminal laser level for us to guess. The ${ }^{4} I_{11 / 2}$ group empties spontaneously by a radiationless phonon transition [2] to the ${ }^{4} I_{9 / 2}$ ground state about $2000 \mathrm{~cm}^{-1}$ below. Measurements with Nd-glass at room temperature show the lifetime of the ${ }^{4} I_{11 / 2}{ }^{4} I_{9 / 2}$ transition likely to be of the order of or greater than $100 \mathrm{~ns}$ [3]. This lifetime and also assumed degeneracies for the terminal laser level have been used as parameters in evaluating measurements of gain saturation for $Q$-switched pulses in $\mathrm{Nd}$-glass amplifiers [4].

Our own measurements have been made with pulses of different durations. When the pulses for amplification were generated by a laser oscillator operating in the random spiking mode with individual spikes longer than a microsecond, our results were in accord with those of 